\title{
TouchStat: A Monte Carlo program for calculating sequential touching probabilities
}

\author{
WALLACE E. DIXON, JR., TIMOTHY WOODARD, and MARK S. MERRY \\ Heidelberg College, Tiffin, Ohio
}

\begin{abstract}
A growing number of child cognition researchers are using an object-manipulation, sequential-touching paradigm to explore young children's conceptual abilities. Within this paradigm, it is essential to distinguish children's intracategory touching sequences from those expected by chance. The sequentialtouching approach is congruent with a permutation testing model of statistical inference and is best modeled by sampled permutations as derived from Monte Carlo procedures. In this article, we introduce a computer program for generating Monte Carlo sequential-touching simulations. TouchStat permits users to determine their own specifications to simulate sequential touching to category exemplars across a wide range of task parameters. We also present Monte Carlo chance probabilities for the standard two-category, four-exemplar task, with sequences up to 30 touches long. Finally, we consider broader applications of the TouchStat program.
\end{abstract}

Over the last decade, there has been an increase in the popularity of spontaneous object-grouping tasks in the assessment of infant categorization (e.g., Bauer, Dow, \& Hertsgaard, 1995; Gopnik \& Meltzoff, 1987, 1992; Mandler \& Bauer, 1988; Mandler, Fivush, \& Reznick, 1987; Shore, Dixon, \& Bauer, 1995). This task usually involves presenting children with a tray containing groups of exemplars from each of two taxonomic categories. The objects are often three-dimensional geometric figures (see, e.g., Gopnik \& Meltzoff, 1987, 1992; Ricciuti, 1965; Sugarman, 1983) or three-dimensional toy replicas of real-world objects (see, e.g., Mandler \& Bauer, 1988; Mandler et al., 1987; Shore et al., 1995), and often differ from each other in one or more perceptual features within a particular category (see, e.g., Bauer et al., 1995). Objects from different superordinate categories such as "animals" and "vehicles" might be contrasted, as might objects from within a single superordinate category, such as "dogs" or "horses" (Bauer et al., 1995; Mandler \& Bauer, 1988). Children are typically not given specific instructions for what to do with the objects, but are simply told, "Here, these are for you to play with," "What can you do with these?" and "Can you fix these up?" (e.g., Bauer et al., 1995; Mandler et al., 1987).

In its original use, the object-grouping task was thought to assess categorical knowledge to the extent that children

Thanks go to Jean Mandler and colleagues for their sequentialtouching Monte Carlo initiative and for graciously making available the results of their Monte Carlo simulations. Special thanks go to Malinda Thomas for running the 785 simulations reported in the Appendix, and to Sandra Waxman and Peg Smith for their encouragement and helpful comments during preparation of the manuscript. Finally, we are grateful for our discussions with Dave Weininger and Hoben Thomas on issues pertaining to probability sampling. Correspondence should be addressed to W. E. Dixon, Jr., Department of Psychology, Heidelberg College, Tiffin, OH 44883 (e-mail: dixon@nike.heidelberg.edu). spatially grouped like objects - that is, objects from within the most inclusive category (Ricciuti, 1965; Starkey, 1981; Sugarman, 1983). One of the limitations of this task has been that younger children do not frequently engage in spontaneous spatial grouping (Sugarman, 1983), although they engage in considerable object manipulation (Oakes, Madole, \& Cohen, 1991; Ruff, 1986). Mandler et al. (1987) addressed this developmental issue by advocating spontaneous sequential intracategory touches as a dependent measure of categorization. Mandler et al. argued that if children "sequentially touch items from the same category more often than would be expected by chance, children must be selecting items on the basis of their relatedness" (p. 348).

\section{PERMUTATION TESTING IN SEQUENTIAL TOUCHING}

The sequential-touching approach falls neatly within a permutation testing model of statistical inference ${ }^{1}$ (Bakeman, Robinson, \& Quera, 1996; May \& Hunter, 1993), although Mandler et al. (1987) do not seem to have recognized this point. In permutation testing, exact probabilities of specific event sequences can be determined with respect to a reference set containing all possible event sequences. Because the reference set comprises all possible event sequences, it effectively becomes the sampling distribution. Moreover, because the entire sampling distribution is known, no inferences about population parameters need to be made. As a special case of permutation testing, analysis of touch sequences is also nonparametric.

This is an important point because if we were to apply traditional "normal curve" statistical models to the sequential-touching procedure, we would be forced to reckon with various parametric assumptions such as whether the total number of touches made by a child is independent of the number of sequential intracategory 
touches. Mandler et al. (1987) appear to have been sensitive to such independence assumptions. After presenting a mathematical equation designed to calculate chance intracategory run length, they wrote, "This equation expresses the fact that chance run length is independent of the total number of touches that are made. Thus it has the advantage of allowing parametric assessment of performance when mean number of touches differs across age groups and across tasks"' (p. 349). However, as Thomas (1995) noted, this assertion is not borne out mathematically. In his parametric modeling of sequential-touching behavior, Thomas has shown that intracategory run length is independent of overall touching only asymptotically, beyond about 10 total touches. The logic of permutation testing obviates preoccupations with independence assumptions because the population of all touch-sequence permutations for a given child is known (Bakeman et al., 1996).

The limiting factor of permutation testing is that construction of the reference sets can be formidable when long or complicated event sequences are involved. For example, there would be $6.20 \times 10^{23}(=n !)$ possible ways a child could touch 24 things. Bakeman et al. (1996) have thus advocated selecting reference set subsamples through Monte Carlo modeling, a procedure they have labeled sampled permutation tests. Sampled permutation tests have the benefit of modeling otherwise extremely impractical permutation distributions; however, because they derive from the permutation testing paradigm, they avoid problems associated with parametric assumptions.

\section{MONTE CARLO MODELING OF SEQUENTIAL TOUCHING}

In an attempt to parse out true category-based touch sequences from otherwise stochastic processes, Mandler et al. (1987) introduced the notion of comparing obtained sequential-touching patterns to computer-generated Monte Carlo touch simulations. This method is of course congruent with the sampled permutation approach described by Bakeman et al. (1996). The idea is straightforward. A child whose intracategory touch sequences exceed sampled permutation test expectations must be selecting intracategorically as a function of category membership. Such children can be labeled categorizers. Furthermore, Mandler et al. argued that, to the extent that such children categorize one or both categories, they can be called single or exhaustive categorizers, respectively.

To model touch sequences, sampled permutation testing must take into account five variables: (1) the number of experimenter-defined categories, (2) the number of items within each category, (3) the total number of child touches made without interruption, (4) the length of any "run" of touches to objects within a single category, and (5) the number of unique objects touched during the intracategory run. The first two variables are fixed by the structure of the task. However, the remaining three variables are determined by the child.

Consider the following example. Suppose a child was presented with eight objects - four from Category A and four from Category B. Suppose further that (1) the child spontaneously touched 20 things (ignoring immediately repeating touches to the same object; see Mandler et al., 1987), (2) within this touch sequence was an uninterrupted run of 10 intracategory touches, and (3) among this 10touch string were touches to at least three unique objects. In this case, the experimenter could compare the child's performance to Monte Carlo touch simulations meeting the same criteria. The probability of occurrence of at least this event is .002 (according to Mandler et al.'s unpublished look-up table). That is, out of 10,000 Monte Carlo sampled permutations, permutations meeting or exceeding these specific criteria happened only 20 times. Because .002 exceeds the conventional 10 cutoff value, the child's behavior is presumed to derive from categorization. Researchers may wish to use more conservative cutoff values such as $p=.05$ or $p=.01$.

Unfortunately, the Mandler et al. (1987) Monte Carlo look-up table is unpublished and hence not widely accessible (although the authors distribute the data freely upon request). More importantly, however, this set of Monte Carlo results applies only to object-manipulation tasks contrasting two categories with four exemplars each. With the increasing popularity of this task and more general applications of the sampled permutation testing procedure, alternative Monte Carlo simulations using different task parameters will likely become necessary. With younger children, one may wish to investigate sequential touching of two categories containing only three members each; with older children, sequential touching with category set sizes greater than four may be explored. Also, if the set size equality constraint is removed, this Monte Carlo procedure can be used to simulate touching behavior in unstructured settings where there may be unequal categorical set sizes, such as mother-child toy selections during free play.

\section{TouchSTAT}

The primary purpose of the present article is to introduce TouchStat, a Monte Carlo program for conducting sampled permutation tests of children's sequentialtouching behaviors. The outputs of TouchStat runs can be used to estimate exact probabilities of specific intracategory touching sequences. A secondary goal of the article is to make widely accessible a Monte Carlo look-up table, produced by TouchStat, using the typical two-category, four-exemplar constraints. However, as already noted, TouchStat is useful beyond the two-category, fourexemplar case because it permits users to specify a range of desired values for all five defining variables. Parameterizing TouchStat involves setting the following variables: 
1. Number of categories: The user can specify the number of categories to be represented in the simulation.

2. Number of items: The user can specify the number of items per category without the constraint that each group contain an equal number of items.

3. Number of total touches: The user can specify the total number of object touches to simulate in each computer-generated sample.

4. Number of touches in a run: The user can specify the number of within-category touches that must occur in sequence.

5. Number of unique items: The user can specify the number of unique objects within a category that must occur within the run.

6. Number of samples: The user can specify the total number of Monte Carlo samples to be drawn, given the above parameters.

\section{MECHANISMS OF TOUCH STAT}

TouchStat was written by T.W. in C and should be compilable on any platform supporting $\mathrm{C}$. The program has been successfully compiled on UNIX, VAX, DEC, MS-DOS, and MacOS platforms. A Windows 95 executable code, utilizing a graphic user interface, is forthcoming (written by M.S.M.). The program is essentially composed of two sets of random number generation and three counters. The generation of an individual Monte Carlo "touch" requires the nesting of the two random number generation subroutines (referred to as the category selector and the item selector for clarity). To simulate a touch, the category selector draws from a pool of values ranging from 1 to the value specified by the Number of Categories parameter. The value returned by the category selector represents the category of the first touch. The item selector then draws from a pool of values ranging from 1 to the value specified by the Number of Items parameter for that particular category. Note that the range of values available to the item selector depends on the output of the category selector. Category 1 might have four items while Category 2 has six items, for example. For each permutation, the program continues "touching" items in this fashion until the number of touches defined by the Number of Total Touches parameter is reached.

The three counters in TouchStat can be referred to as the run counter, the uniqueness counter, and the tallier. For each sampled permutation of $N$ total touches, the run counter monitors sequences of intracategory touches. If the run length of a given string of intracategory touches equals the value specified in the Number of Touches in a Run parameter, the run counter sets to true. The uniqueness counter monitors the number of unique items selected within a run. Researchers have conventionally required that at least three unique items be selected during any string of intracategory touches (e.g., Mandler et al., 1987), but alternative criteria can be applied. To apply the conventional criterion, the Number of Unique Items parameter would be set to 3 . If at least three unique items are touched during the run, the uniqueness counter sets to true. Finally, at the completion of each sampled permutation, the tallier checks to see if both the run counter and the uniqueness counter are true. If so, the tallier advances. When output files are used, the tallier marks instances in which special cases occur. After TouchStat completes its Monte Carlo "touching," the tallier value is divided by the number of sampled permutations generated. The resulting ratio represents an estimate of the exact probability of the target touch sequence, given the reference set of all possible permutations (see Bakeman et al., 1996).

\section{SPECIFYING TOUCHSTAT PARAMETERS}

Table 1 presents a sample dialogue between TouchStat and a user in which a typical set of specifications is provided. First, the user specifies that the output be displayed in a file. ${ }^{2}$ In the Category Data Section, the user parameterizes TouchStat to simulate presentation of a "tray" containing four exemplars from each of two categories. In the Sample Data section, the user specifies the sampling constraints-in this instance, 20 total touches to be simulated 30 times. In the Special Cases section, the user defines one special case of seven intracategory touches to at least three unique items from one of the two categories. The Results section returns the number of special cases produced as a percentage of the total number of sampled permutations - in this case, 1 out of 30 .

\section{INTERPRETING THE OUTPUT}

A sample output generated by TouchStat, accompanied by interpretations of the output values, can be found in Table 2. These results are from an actual run produced by TouchStat using the settings just described. The hit run and a sample miss run are presented. Of course, 20 touches were generated in each case. The hit run is counted as such because the user-defined special case conditions were met: (1) There were at least seven sequential touches to Category 1 exemplars, and (2) there were at least three unique exemplars touched within the seventouch sequence. Note that in this particular permutation sample, TouchStat actually generated an intracategory touch string as long as nine touches, which happened to include touches to all four unique exemplars. The program counts as special cases all cases that meet or exceed the specified criteria. For reference, the Appendix presents the Monte Carlo probabilities derived from 100,000 sampled permutations for each of $N=3$ to 30 total touches in the standard two-category, four-exemplar paradigm, including touches to both three and four unique objects.

\section{SPECIAL FEATURES}

TouchStat provides several useful features in addition to permitting user-determined settings. For example, 
Table 1

Sample Dialogue From TouchStat

\begin{tabular}{|c|c|}
\hline TouchStat v1.1 - 1996 Tim Woodard & ***Version and author of TouchStat \\
\hline Output run data $(y / n): y$ & $\begin{array}{l}\text { *** Do you want the results of the } \\
\text { *** Monte Carlo run to be displayed? }\end{array}$ \\
\hline Output to file, screen, or both $(\mathrm{f} / \mathrm{s} / \mathrm{b})$ : $\mathrm{f}$ & ***How do you want it displayed? \\
\hline \multicolumn{2}{|l|}{$* * * * * * * * * * * * *$ Category Data $* * * * * * * * * * * * *$} \\
\hline Number of Categories: 2 & $\begin{array}{l}* * * \text { How many categories do you want } \\
* * * \text { to simulate? }\end{array}$ \\
\hline Number of Items in Cat & *** How many ite \\
\hline Number & $* * *$ do \\
\hline \multicolumn{2}{|l|}{$* * * * * * * * * * * * * *$ Sample Data $* * * * * * * * * * * * * *$} \\
\hline Number of Total Touches in a Sample: 20 & $\begin{array}{l}* * * \text { How many total touches to objects } \\
* * * \text { on the tray do you want to } \\
* * * \text { simulate? }\end{array}$ \\
\hline Number of Samples: 30 & $\begin{array}{l}{ }^{* * *} \text { How many Monte Carlo runs with } \mathrm{x} \\
{ }^{* * *} \text { touches do you want to simulate? }\end{array}$ \\
\hline \multicolumn{2}{|l|}{$* * * * * * * * * * * *$ Special Cases $* * * * * * * * * * *$} \\
\hline Number of Special Cases: 1 & $\begin{array}{l}* * * \text { How many specific touch sequences } \\
* * * \text { do you want to look for? }\end{array}$ \\
\hline Number of Touches in Case[1]: 7 & $\begin{array}{l}* * * \text { In this specific touch sequence, } \\
* * * \text { how many intracategory touches in } \\
* * * \text { sequence do you want to look for? }\end{array}$ \\
\hline Number of Unique Items: 3 & $\begin{array}{l}\text { ***How many unique objects in this } \\
* * * \text { touch sequence should be touched? }\end{array}$ \\
\hline Category of Case[1] ( 0 for any): 1 & $\begin{array}{l}* * * \text { To which category should the } \\
\text { ***touches be directed? }\end{array}$ \\
\hline \multicolumn{2}{|l|}{$* * * * * * * * * * * * * * *$ Results $* * * * * * * * * * * * * *$} \\
\hline $\begin{array}{l}\text { Computing probability: }\|\|\|\| \\
\text { Probability of special case: } \% 3.333333(1: 30)\end{array}$ & $\begin{array}{l}* * * \text { Progress bar. } \\
* * * \# \text { of hits out of \# of runs. }\end{array}$ \\
\hline
\end{tabular}

when conducting successive Monte Carlo simulations, TouchStat permits retention of the same category and item data. This feature is advantageous when, for a given set of task constraints, the behaviors of a number of individual children need to be modeled.

TouchStat also permits users to look for more than one special case in a given permutation sample. For example, the program can determine the chance probability in a single permutation sample of obtaining both a Category 1 three-unique-item, 11 -intracategory-touch sequence, and a Category 2 four-unique-item, nine-intracategorytouch sequence. Such special cases can be constrained to occur in a particular order or any order.

\section{BEYOND THE SEQUENTIAL-TOUCHING TASK}

TouchStat was designed specifically to evaluate the chance touching behaviors of young children in a sequential-touching task. However, as a generalized permutation sampling program, it can be parameterized to conduct sampled permutation tests in any number of alternative settings in which sequential selections are thought to be based on category membership. What follows are some possible alternative applications of
TouchStat-based Monte Carlo simulations in successively more general contexts.

\section{Determining Salience-Based Categorizing}

The utility of applying sampled permutation testing to the issue of children's categorization has already been demonstrated. However, the theoretically contentious issue of how children are making their object selections has been sidestepped. Researchers within the Mandlerian paradigm generally assume that object selections are based on conceptual category membership - that is, the extent to which objects belong to one or another taxonomic or thematic concept. Yet it could be argued that children categorize on the basis of perceptual features, the most salient of which happen to belong to one class. But, as Mandler et al. (1987) pointed out, "This argument is cogent only for the case in which the exemplars are identical ... The argument becomes considerably less cogent when the categories consist of [perceptually] dissimilar items" (p. 348).

To be sure, intracategory objects cannot avoid sharing some perceptual features (Rosch, Mervis, Gray, Johnson, \& Boyes-Braem, 1976). But because of the potential confounding of perceptual salience with category membership, experimenters should also take care in selecting perceptually diverse exemplars within each cate- 
Table 2

Sample Output From TouchStat

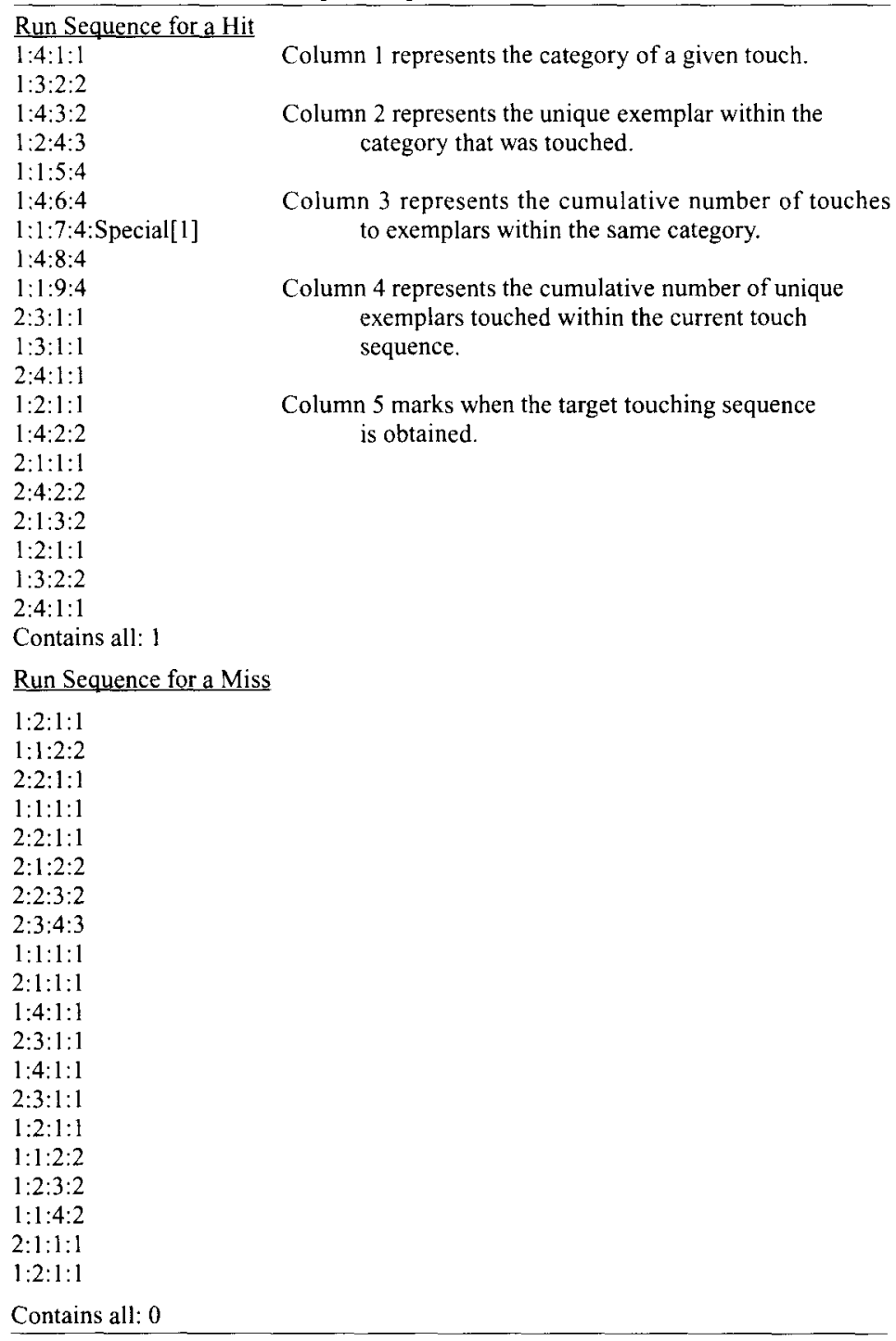

gory. Under these circumstances, the problem of perceptual salience becomes empirically addressable through sampled permutation testing. If the perceptual salience of a set of exemplars can be determined a priori on the basis of some independent criteria, researchers can redefine the objects as belonging to classes of salient and nonsalient things. Or (because TouchStat is not limited to the two-group case) perhaps the objects could be divided into highly salient, moderately salient, and nonsalient groups. If perceptual diversity within classes is high, perceptual salience will no doubt transcend the original conceptual boundaries. Children's touching patterns to these new perceptual-salience-based categories could then be analyzed following the standard procedures.

\section{Category-Based Object Selection in Free Play}

TouchStat can also be used to sample permutations outside the domain of the sequential-touching task. For example, researchers are often interested in examining the play behaviors of children or parents during parentinfant free-play sessions (e.g., Tamis-LeMonda \& Bornstein, 1994). In free play, any number of toys or types of toy might serve as a vehicle for higher or extended levels of play (Dixon \& Shore, 1993). TouchStat could be used in such an instance to determine whether toys are selected for play as a function of a theoretically relevant attribute. Toy attributes of interest might include conceptual or perceptual similarity, but might also include gender appropriateness or ability to facilitate pretend play.

An issue of recent concern in the literature is the extent to which parents provide supportive play environments for their children (Dixon \& Shore, 1993; TamisLeMonda \& Bornstein, 1994). This question might be rephrased to address the extent to which parents select play objects that are appropriate to their children's level of cognitive development. One way to approach this issue 
is from the perspective of permutation testing. Suppose a researcher were interested in determining whether parents are more likely to select a series of pretend-facilitating toys during free play than would be expected by chance. The available free-play toys could be divided into those supporting nonsymbolic play (e.g., blocks, ball, popbeads) and those supporting symbolic play (e.g., phone, teddy bear, bowl and spoon). In this instance, the number of exemplars in each category of toy would likely differa situation ideally suited for TouchStat.

To model random toy selection using TouchStat, then, the researcher would set the Number of Categories variable to 2 and the Number of Items variable to the appropriate value for each of the two (nonsymbolic and symbolic) categories. The values of the remaining variables would depend on the specific parental toy selections to be modeled, such as the total number of toys selected and the number of symbolic-play-supporting toys selected in sequence. TouchStat would proceed to sample from all possible permutations given these constraints. The probability of a given parent's toy selection behavior would be returned, taking into account toy availability base rates.

\section{Applications Outside the Developmental Area}

Applications for TouchStat outside the developmental area are also possible. In group decision making, for example, a researcher could determine the probabilities of specific individuals making sequences of eye contact with self-defined allies, foes, or neutral individuals, relative to chance expectations. In this situation, the Number of Categories parameter would be set at 3 , and the Number of Items parameter in each category would change as a function of the participant's individual "foe-alliance" perceptions. Psychotherapy process researchers might wish to investigate whether specific types of client and/or therapist are biased toward using a series of main clause cognitive verbs relative to verbs of affect (Gervasio, 1984), or any number of categories of verb. Chance probabilities of verb-use sequences in this case would be calculated as a function of the total number of main-clause verbs produced in a given psychotherapeutic session.

TouchStat parameters can also be defined to simulate typical random event sequences such as coin tosses or dice throws. To simulate coin tosses, for example, the Number of Categories parameter should be set to 2, with one item in each category. Here, Category 1 touches could represent "heads" and Category 2 touches could represent "tails." The Number of Total Touches parameter in each sample should be set to 1 , as should the number of special cases, the number of touches in Special Case 1, the number of unique items, and the category of Special Case 1. Using these values, and by specifying 1 million samples (coin tosses) on three consecutive trials, TouchStat produced "heads" 499,821, 500,012, and 500,206 times, respectively. Dice throws can be similarly modeled, using six categories with one item each. Three trials of 1 million dice throw samples yielded target values of 166,696 ,
165,876 , and 166,837 times, respectively, with an expected value of 166,667 times. $^{3}$

\section{CONCLUSIONS}

Researchers employing the sequential-touching task have made considerable progress toward revealing the nature of children's cognitive and conceptual development. Of course, methodologies from a variety of alternative paradigms also have been fruitful in the investigation of categorical development. Waxman and her colleagues have made significant contributions toward understanding taxonomic versus thematic conceptualization beyond the 1st year, using a forced-choice procedure (Waxman \& Hall, 1993; Waxman \& Namy, 1997). Many researchers have combined object-manipulation and visual-habituation techniques to investigate conceptual development in children as young as 6 or 7 months of age (Mandler \& McDonough, 1993; Oakes et al., 1991; Ross, 1980; Ruff, 1986; Waxman \& Markow, 1995), and research employing a visual habituation-dishabituation paradigm points to at least perceptual categorization in even very early infancy (see, e.g., Colombo \& Mitchell, 1990). Continued progress toward a more complete understanding of children's categorical development depends on methodological contributions from all these paradigms. We believe that analysis of children's sequentialtouching behaviors from within a sampled permutation testing context, implementable through TouchStat, provides a profitable addition to an already powerful arsenal of methodological innovations.

\section{Availability}

Touchstat is available through direct download free of charge. Floppy disk versions are available for $\$ 3.50$ (cost of disk and postage). For either version, contact W.E.D. at dixon@nike.heidelberg.edu).

\section{REFERENCES}

Bakeman, R., Robinson, B. F., \& Quera, V. (1996). Testing sequential association: Estimating exact $p$ values using sampled permutations. Psychological Methods, 1, 4-15.

Bauer, P. J., Dow, G. A., \& HertsgaARd, L. A. (1995). Effects of prototypicality on categorization in 1- to 2-year-olds: Getting down to basic. Cognitive Development, 10, 43-68.

Colombo, J., \& Mitchell, D. W. (1990). Individual differences in early visual attention: Fixation time and information processing. In J. Colombo \& J. Fagen (Eds.), Individual differences in infancy: Reliability, stability, prediction (pp. 193-227). Hillsdale, NJ: Erlbaum.

Dixon, W. E., JR., \& Shore, C. (1993). What shall we play? Play content as a vehicle for mother--infant interaction. Infant Behavior \& Development, 16, 121-125.

Gervasio, A. H. (1984). Computer-assisted analysis of conversation. Behavior Research Methods, Instruments, \& Computers, 16, 158-161.

Gopnik, A., \& MELtzoff, A. (1987). The development of categorization in the 2 nd year and its relation to other cognitive and linguistic developments. Child Development, 58, 1523-1531.

Gopnik, A., \& Meltzoff, A. (1992). Categorization and naming Basic-level sorting in 18 -month-olds and its relation to language Child Development, 63, $1091-1103$.

Mandler, J. M., \& Bauer, P. J. (1988). The cradle of categorization: Is the basic level basic? Cognitive Development, 3, 247-264. 
Mandler, J. M., Fivush, R., \& Reznick, J. S. (1987). The development of contextual categories. Cognitive Development, 2, 339-354.

Mandler, J. M., \& McDonough, L. (1993). Concept formation in infancy. Cognitive Development, 8, 291-318.

MAY, R. B., \& HUNTER, M. A. (1993). Some advantages of permutation tests. Canadian Psychology, 34, 401-407.

OAKes, L. M., Madole, K. L., \& Cohen, L. B. (1991). Infants' object examining: Habituation and categorization. Cognitive Development, 6, 377-392.

RICCIUTI, H. (1965). Object grouping and selective ordering behavior in infants 12 to 24 months old. Merrill-Palmer Quarterly, 11, 129-148.

Rosch, E., Mervis, C., Gray, W., Johnson, D., \& Boyes-Braem, P. (1976). Basic objects in natural categories. Cognitive Psychology, 8 , 382-439.

Ross, G. S. (1980). Categorization in 1- to 2-year-olds. Developmental Psychology, 16, 391-396.

RUFF, H. (1986). Components of attention during infants' manipulative exploration. Child Development, 57, 105-114.

Shore, C., DiXon, W. E., JR., \& Bauer, P. J. (1995). Measures of linguistic and non-linguistic knowledge of objects in the 2nd year. First Language, 15, 189-202.

STARKEY, D. (1981). The origins of concept formation: Object sorting and object preference and early infancy. Child Development, 52, 489-497.

Sugarman, S. (1983). Children 's early thought: Developments in classification. Cambridge, MA: Cambridge University Press.

Tamis-LeMonda, C. S., \& Bornstein, M. H. (1994). Specificity in mother-toddler language-play relations across the 2 nd year. Developmental Psychology, 30, 283-292.

Thомаs, H. (1995). On Mandler, Fivush, and Reznick's analysis of infant categorization data. Unpublished manuscript, Pennsylvania State University.

WAXMAN, S. R., \& Hall, D. G. (1993). The development of a linkage between count nouns and object categories: Evidence from 15- to 21month-old infants. Child Development, 64, 1224-1241.

WAXMAN, S. R., \& MARKOW, D. B. (1995). Words as invitations to form categories: Evidence from 12- to 13-month-old infants. Cognitive Psychology, 29, 257-302.

WAXMAN, S. R., \& NAMY, L. L. (1997). Challenging the notion of a thematic preference in young children. Developmental Psychology, 33, 555-567.

\section{NOTES}

1. I thank Roger Bakeman for bringing this point to my attention. 2 . The display option (particularly when the user is selecting display to screen) should be employed only when very small numbers of samples are desired. Large sample simulations will produce formidably large files and will take considerably longer to be generated.

3. The coin toss and dice throw simulations can also serve as a check on the "randomness" of the random number generation subroutines, which, in this case, were conducted on a Sun SPARCserver 670/MP (UNIX). 
APPENDIX

Proportion of Special Cases in One Category ( $N=$ number of total touches)

\begin{tabular}{|c|c|c|c|c|c|c|c|c|c|c|}
\hline & \multicolumn{10}{|c|}{$N$} \\
\hline & \multicolumn{2}{|c|}{3} & \multicolumn{2}{|c|}{4} & \multicolumn{2}{|c|}{5} & \multicolumn{2}{|c|}{6} & \multicolumn{2}{|c|}{7} \\
\hline & 3 Unique & 4 Unique & 3 Unique & 4 Unique & 3 Unique & 4 Unique & 3 Unique & 4 Unique & 3 Unique & 4 Unique \\
\hline 3 & .0616 & $\mathrm{n} / \mathrm{a}$ & .0971 & $\mathrm{n} / \mathrm{a}$ & .1311 & $\mathrm{n} / \mathrm{a}$ & .1651 & $\mathrm{n} / \mathrm{a}$ & 1989 & $\mathrm{n} / \mathrm{a}$ \\
\hline 4 & & & .0341 & .0090 & .0550 & .0137 & .0761 & .0184 & .0949 & .0232 \\
\hline 5 & & & & & .0160 & .0076 & .0263 & .0116 & .0355 & .0159 \\
\hline 6 & & & & & & & .0067 & .0047 & .0154 & .0071 \\
\hline 7 & & & & & & & & & .0031 & .0024 \\
\hline 8 & & & & & & & & & & \\
\hline 9 & & & & & & & & & & \\
\hline 10 & & & & & & & & & & \\
\hline 11 & & & & & & & & & & \\
\hline 12 & & & & & & & & & & \\
\hline 13 & & & & & & & & & & \\
\hline 14 & & & & & & & & & & \\
\hline 15 & & & & & & & & & & \\
\hline 16 & & & & & & & & & & \\
\hline 17 & & & & & & & & & & \\
\hline 18 & & & & & & & & & & \\
\hline 19 & & & & & & & & & & \\
\hline 20 & & & & & & & & & & \\
\hline 21 & & & & & & & & & & \\
\hline 22 & & & & & & & & & & \\
\hline 23 & & & & & & & & & & \\
\hline 24 & & & & & & & & & & \\
\hline 25 & & & & & & & & & & \\
\hline 26 & & & & & & & & & & \\
\hline 27 & & & & & & & & & & \\
\hline 28 & & & & & & & & & & \\
\hline 29 & & & & & & & & & & \\
\hline 30 & & & & & & & & & & \\
\hline
\end{tabular}


APPENDIX (Continued)

\begin{tabular}{|c|c|c|c|c|c|c|c|c|c|}
\hline \multicolumn{10}{|c|}{$N$} \\
\hline \multicolumn{2}{|c|}{8} & \multicolumn{2}{|c|}{9} & \multicolumn{2}{|c|}{10} & \multicolumn{2}{|c|}{11} & \multicolumn{2}{|c|}{12} \\
\hline 3 Unique & 4 Unique & 3 Unique & 4 Unique & 3 Unique & 4 Unique & 3 Unique & 4 Unique & 3 Unique & 4 Unique \\
\hline .2307 & $\mathrm{n} / \mathrm{a}$ & .2632 & $\mathrm{n} / \mathrm{a}$ & .2882 & $\mathrm{n} / \mathrm{a}$ & .3174 & $\mathrm{n} / \mathrm{a}$ & .3418 & $\mathrm{n} / \mathrm{a}$ \\
\hline .1144 & .0289 & .1335 & .0343 & .1546 & .0370 & .1698 & .0434 & .1897 & .0475 \\
\hline .0448 & .0196 & .0537 & .0242 & .0621 & .0292 & .0722 & .0334 & .0805 & .0370 \\
\hline .0159 & .0096 & .0189 & .0123 & .0231 & .0147 & .0284 & .0186 & .0323 & .0198 \\
\hline .0049 & .0039 & .0066 & .0047 & .0082 & .0062 & .0109 & .0075 & .0120 & .0083 \\
\hline \multirow[t]{5}{*}{.0016} & .0010 & .0020 & .0017 & .0026 & .0021 & .0034 & .0029 & .0049 & .0036 \\
\hline & & .0007 & .0005 & .0009 & .0010 & .0012 & .0010 & .0016 & .0014 \\
\hline & & & & .0003 & .0003 & .0004 & .0003 & .0005 & .0004 \\
\hline & & & & & & .0001 & .00005 & .0001 & .0002 \\
\hline & & & & & & & & .00001 & .00007 \\
\hline
\end{tabular}


APPENDIX (Continued)

\begin{tabular}{|c|c|c|c|c|c|c|c|c|c|c|}
\hline & & & & & & & & & & \\
\hline & & & & & & & & & & \\
\hline & 3 Unique & 4 Unique & 3 Unique & 4 Unique & 3 Unique & 4 Unique & 3 Unique & 4 Unique & 3 Unique & 4 Unique \\
\hline 3 & .3678 & $\mathrm{n} / \mathrm{a}$ & .3915 & $\mathrm{n} / \mathrm{a}$ & .4179 & $\mathrm{n} / \mathrm{a}$ & .4412 & $\mathrm{n} / \mathrm{a}$ & .46323 & $\mathrm{n} / \mathrm{a}$ \\
\hline 4 & .2054 & .0544 & .2258 & .0577 & .2253 & .0625 & .2573 & .06629 & .27386 & .07196 \\
\hline 5 & .0888 & .0418 & .0986 & .0453 & .1071 & .0489 & .1150 & .05419 & .12624 & .05776 \\
\hline 6 & .0361 & .0227 & .0399 & .0259 & .0441 & .0264 & .0483 & .02984 & .05209 & .03285 \\
\hline 7 & .0142 & .0102 & .0152 & .0118 & .0169 & .0125 & .0191 & .01444 & .02035 & .01577 \\
\hline 8 & .0052 & .0041 & .0061 & .0050 & .0066 & .0054 & .0073 & .00596 & .00808 & .00692 \\
\hline 9 & .0017 & .0016 & .0022 & .0020 & .0026 & .0023 & .0027 & .00224 & .00319 & .00303 \\
\hline 10 & .0006 & .0006 & .0009 & .0008 & .0008 & .0009 & .0010 & .00107 & .00116 & .00096 \\
\hline 11 & .0003 & .0003 & .0003 & .0003 & .0003 & .0003 & .0003 & .00042 & .00045 & .00044 \\
\hline 12 & .00012 & .00011 & .0001 & .0001 & .0002 & .0001 & .00016 & .00017 & .00019 & .00018 \\
\hline 13 & .00001 & .00002 & .00007 & .00003 & .00004 & .00008 & .00007 & .00002 & .00009 & .00012 \\
\hline 14 & & & 0 & .00001 & .00003 & .00001 & .00003 & .00002 & .00001 & .00002 \\
\hline 15 & & & & & 0 & 0 & 0 & 0 & 0 & 0 \\
\hline 16 & & & & & & & 0 & 0 & 0 & 0 \\
\hline 17 & & & & & & & & & 0 & 0 \\
\hline 18 & & & & & & & & & & \\
\hline 19 & & & & & & & & & & \\
\hline 20 & & & & & & & & & & \\
\hline 21 & & & & & & & & & & \\
\hline 22 & & & & & & & & & & \\
\hline 23 & & & & & & & & & & \\
\hline 24 & & & & & & & & & & \\
\hline 25 & & & & & & & & & & \\
\hline 26 & & & & & & & & & & \\
\hline 27 & & & & & & & & & & \\
\hline 28 & & & & & & & & & & \\
\hline 29 & & & & & & & & & & \\
\hline 30 & & & & & & & & & & \\
\hline
\end{tabular}


APPENDIX (Continued)

\begin{tabular}{|c|c|c|c|c|c|c|c|c|c|}
\hline \multicolumn{10}{|c|}{$N$} \\
\hline \multicolumn{2}{|c|}{18} & \multicolumn{2}{|c|}{19} & \multicolumn{2}{|c|}{20} & \multicolumn{2}{|c|}{21} & \multicolumn{2}{|c|}{22} \\
\hline 3 Unique & 4 Unique & 3 Unique & 4 Unique & 3 Unique & 4 Unique & 3 Unique & 4 Unique & 3 Unique & 4 Unique \\
\hline .48531 & $\mathrm{n} / \mathrm{a}$ & .50532 & $\mathrm{n} / \mathrm{a}$ & .52198 & $\mathrm{n} / \mathrm{a}$ & .54399 & $n / a$ & .56179 & $\mathrm{n} / \mathrm{a}$ \\
\hline .29063 & .07824 & .30422 & .08242 & .32066 & .08667 & .33764 & .09101 & .34972 & .09698 \\
\hline .13223 & .06370 & .14166 & .06785 & .07103 & .07035 & .15819 & .07524 & .16683 & .07886 \\
\hline .05520 & .03502 & .05885 & .03800 & .06558 & .04032 & .06655 & .04241 & .07256 & .04368 \\
\hline .02256 & .01670 & .02363 & .01841 & .02601 & .01861 & .02762 & .02027 & .02913 & .02175 \\
\hline .00836 & .00758 & .00930 & .00758 & .01083 & .00856 & .01107 & .00920 & .01177 & .00988 \\
\hline .00403 & .00312 & .00424 & .00308 & .00402 & .00356 & .00468 & .00404 & .00481 & .00415 \\
\hline .00137 & .00125 & .00150 & .00120 & .00153 & .00171 & .00159 & .00158 & .00166 & .00182 \\
\hline .00049 & .00054 & .00050 & .00060 & .00066 & .00077 & .00082 & .00046 & .00073 & .00059 \\
\hline .00020 & .00028 & .00022 & .00015 & .00037 & .00023 & .00022 & .00030 & .00031 & .00027 \\
\hline .00011 & .00009 & .00013 & .00004 & .00012 & .00008 & .00006 & .00010 & .00005 & .00012 \\
\hline 0 & .00001 & .00002 & .00007 & .00006 & .00006 & .00006 & .00003 & .00008 & .00005 \\
\hline .00002 & .00001 & 0 & 0 & .00002 & 0 & .00002 & .00002 & .00002 & .00001 \\
\hline 0 & 0 & .00001 & .00001 & 0 & 0 & .00001 & .00001 & .00001 & 0 \\
\hline 0 & 0 & 0 & 0 & 0 & 0 & 0 & 0 & 0 & .00003 \\
\hline \multirow[t]{5}{*}{0} & 0 & 0 & 0 & 0 & 0 & 0 & 0 & 0 & 0 \\
\hline & & 0 & 0 & 0 & 0 & 0 & 0 & 0 & 0 \\
\hline & & & & 0 & 0 & 0 & 0 & 0 & 0 \\
\hline & & & & & & 0 & 0 & 0 & 0 \\
\hline & & & & & & & & 0 & 0 \\
\hline
\end{tabular}


APPENDIX (Continued)

\begin{tabular}{|c|c|c|c|c|c|c|c|c|c|c|}
\hline & \multicolumn{10}{|c|}{$N$} \\
\hline & \multicolumn{2}{|c|}{23} & \multicolumn{2}{|c|}{24} & \multicolumn{2}{|c|}{25} & \multicolumn{2}{|c|}{26} & \multicolumn{2}{|c|}{27} \\
\hline & 3 Unique & 4 Unique & 3 Unique & 4 Unique & 3 Unique & 4 Unique & 3 Unique & 4 Unique & 3 Unique & 4 Unique \\
\hline 3 & .57900 & $\mathrm{n} / \mathrm{a}$ & .59376 & $\mathrm{n} / \mathrm{a}$ & .61088 & $\mathrm{n} / \mathrm{a}$ & .62435 & $\mathrm{n} / \mathrm{a}$ & .64105 & $\mathrm{n} / \mathrm{a}$ \\
\hline 4 & .36322 & .09976 & .37803 & .10570 & .39055 & .10996 & .40308 & .11465 & .41805 & .11837 \\
\hline 5 & .17417 & .08171 & .18143 & .08760 & .18967 & .09076 & .20107 & .09415 & .20774 & .09974 \\
\hline 6 & .07352 & .04536 & .08021 & .07889 & .08295 & .05326 & .08587 & .05416 & .08905 & .05577 \\
\hline 7 & .03179 & .02383 & .03202 & .02475 & .03430 & .03391 & .03545 & .02641 & .03800 & .02864 \\
\hline 8 & .01287 & .01052 & .01328 & .01103 & .01430 & .01072 & .01508 & .01263 & .01525 & .01277 \\
\hline 9 & .00481 & .00475 & .00486 & .00451 & .00598 & .00487 & .00601 & .00557 & .00660 & .00537 \\
\hline 10 & .00189 & .00178 & .00234 & .00233 & .00225 & .00230 & .00242 & .00223 & .00240 & .00233 \\
\hline 11 & .00106 & .00090 & .00112 & .00107 & .00095 & .00082 & .00093 & .00096 & .00112 & .00104 \\
\hline 12 & .00039 & .00034 & .00036 & .00039 & .00040 & .00039 & .00040 & .00037 & .00042 & .00042 \\
\hline 13 & .00012 & .00016 & .00015 & .00013 & .00012 & .00026 & .00009 & .00016 & .00020 & .00013 \\
\hline 14 & .00005 & .00005 & .00007 & .00004 & .00009 & .00006 & .00006 & .00012 & .00007 & .00007 \\
\hline 15 & .00002 & .00002 & .00001 & 0 & .00003 & .00005 & .00002 & .00002 & .00002 & .00001 \\
\hline 16 & 0 & 0 & 0 & .00002 & 0 & 0 & 0 & 0 & .00001 & .00002 \\
\hline 17 & 0 & 0 & 0 & .00002 & 0 & 0 & .00001 & .00001 & .00001 & .00001 \\
\hline 18 & 0 & 0 & 0 & 0 & 0 & 0 & 0 & 0 & 0 & 0 \\
\hline 19 & 0 & 0 & 0 & 0 & 0 & 0 & .00001 & 0 & 0 & 0 \\
\hline 20 & 0 & 0 & 0 & 0 & 0 & 0 & 0 & 0 & 0 & 0 \\
\hline 21 & 0 & 0 & 0 & 0 & 0 & 0 & 0 & 0 & 0 & 0 \\
\hline 22 & 0 & 0 & 0 & 0 & 0 & 0 & 0 & 0 & 0 & 0 \\
\hline 23 & 0 & 0 & 0 & 0 & 0 & 0 & 0 & 0 & 0 & 0 \\
\hline 24 & & & 0 & 0 & 0 & 0 & 0 & 0 & 0 & 0 \\
\hline 25 & & & & & 0 & 0 & 0 & 0 & 0 & 0 \\
\hline 26 & & & & & & & 0 & 0 & 0 & 0 \\
\hline 27 & & & & & & & & & 0 & 0 \\
\hline 28 & & & & & & & & & & \\
\hline 29 & & & & & & & & & & \\
\hline 30 & & & & & & & & & & \\
\hline
\end{tabular}


APPENDIX (Continued)

\begin{tabular}{|c|c|c|c|c|c|}
\hline \multicolumn{6}{|c|}{$N$} \\
\hline \multicolumn{2}{|c|}{28} & \multicolumn{2}{|c|}{29} & \multicolumn{2}{|c|}{30} \\
\hline 3 Unique & 4 Unique & 3 Unique & 4 Unique & 3 Unique & 4 Unique \\
\hline .65525 & $\mathrm{n} / \mathrm{a}$ & .66938 & $\mathrm{n} / \mathrm{a}$ & .68326 & $\mathrm{n} / \mathrm{a}$ \\
\hline .42946 & .12390 & .44265 & .12791 & .45514 & .13337 \\
\hline .21278 & .10216 & .22249 & .10579 & .22927 & .11021 \\
\hline .09509 & .05871 & .09832 & .06302 & .10359 & .06582 \\
\hline .04007 & .02916 & .04220 & .03041 & .04350 & .03237 \\
\hline .01719 & .01440 & .01743 & .01456 & .01756 & .01532 \\
\hline .00620 & .00600 & .00723 & .00618 & .00735 & .00631 \\
\hline .00281 & .00244 & .00288 & .00250 & .00306 & .00282 \\
\hline .00112 & .00093 & .00141 & .00119 & .00123 & .00131 \\
\hline .00052 & .00034 & .00051 & .00042 & .00051 & .00050 \\
\hline .00017 & .00020 & .00014 & .00014 & .00021 & .00017 \\
\hline .00006 & .00006 & .00004 & .00011 & .00008 & .00003 \\
\hline .00003 & .00004 & .00004 & .00001 & .00004 & .00005 \\
\hline .00001 & 0 & 0 & .00002 & 00002 & 0 \\
\hline .00001 & 0 & 0 & 0 & 0 & .00002 \\
\hline .00001 & 0 & 0 & 0 & 0 & 0 \\
\hline 0 & 0 & 0 & 0 & 0 & 0 \\
\hline 0 & 0 & 0 & 0 & 0 & 0 \\
\hline 0 & 0 & 0 & 0 & 0 & 0 \\
\hline 0 & 0 & 0 & 0 & 0 & 0 \\
\hline 0 & 0 & 0 & 0 & 0 & 0 \\
\hline 0 & 0 & 0 & 0 & 0 & 0 \\
\hline 0 & 0 & 0 & 0 & 0 & 0 \\
\hline 0 & 0 & 0 & 0 & 0 & 0 \\
\hline 0 & 0 & 0 & 0 & 0 & 0 \\
\hline \multirow[t]{3}{*}{0} & 0 & 0 & 0 & 0 & 0 \\
\hline & & 0 & 0 & 0 & 0 \\
\hline & & & & 0 & 0 \\
\hline
\end{tabular}

(Manuscript received March 14, 1997;

revision accepted for publication October 14, 1997.) 